\title{
Ultrafast nonequilibrium dynamics of strongly coupled resonances in the intrinsic cavity of $\mathrm{WS}_{2}$ nanotubes
}

\author{
Bojana Višić $\left(1,{ }^{1,2,3, *}\right.$ Lena Yadgarov, ${ }^{1}$ Eva A. A. Pogna, ${ }^{4}$ Stefano Dal Conte, ${ }^{4}$ Victor Vega-Mayoral, $, 6,7$ Daniele Vella, ${ }^{5,6,8,9}$ \\ Reshef Tenne, ${ }^{1}$ Giulio Cerullo, ${ }^{4}$ and Christoph Gadermaier ${ }^{4,5,6,10, \dagger}$ \\ ${ }^{1}$ Department of Materials and Interfaces, Weizmann Institute of Science, Rehovot 76100, Israel \\ ${ }^{2}$ Department of Condensed Matter Physics, Jozef Stefan Institute, Jamova 39, 1000 Ljubljana, Slovenia \\ ${ }^{3}$ Center for Solid State Physics and New Materials, Institute of Physics Belgrade, University of Belgrade, \\ Pregrevica 118, 11080 Belgrade, Serbia \\ ${ }^{4}$ Department of Physics, Politecnico di Milano, P. Leonardo da Vinci 32, 20133 Milan, Italy \\ ${ }^{5}$ Department of Complex Matter, Jozef Stefan Institute, Jamova 39, 1000 Ljubljana, Slovenia \\ ${ }^{6}$ Jozef Stefan International Postgraduate School, Jamova 39, 1000 Ljubljana, Slovenia \\ ${ }^{7}$ School of Physics, Trinity College Dublin, Dublin 2, Ireland \\ ${ }^{8}$ Department of Physics, National University of Singapore, 2 Science Drive 3, Singapore 117542, Singapore \\ ${ }^{9}$ Faculty of Mechanical Engineering, University of Ljubljana, Askerceva 6, 1000 Ljubljana, Slovenia \\ ${ }^{10}$ Center for Nano Science and Technology, Italian Institute of Technology, V. Pascoli 70/3, 20133 Milano, Italy
}

(Received 19 November 2018; revised manuscript received 28 May 2019; published 24 October 2019)

\begin{abstract}
Strong coupling of electric transition dipoles with optical or plasmonic resonators modifies their light-matter interaction and, therefore, their optical spectra. Semiconducting $\mathrm{WS}_{2}$ nanotubes intrinsically provide the dipoles through their excitonic resonances, and the optical cavity via their cylindrical shape. We investigate the nonequilibrium light-matter interaction in $\mathrm{WS}_{2}$ nanotubes in the time domain using femtosecond transient extinction spectroscopy. We develop a phenomenological coupled oscillator model with time-dependent parameters to describe the transient extinction spectra, allowing us to extract the underlying nonequilibrium electron dynamics. We find that the exciton and trion resonances shift due to many-body effects of the photogenerated charge carriers and their population dynamics on the femto- and picosecond timescale. Our results show that the time-dependent phenomenological model quantitatively reproduces the nonequilibrium optical response of strongly coupled systems.
\end{abstract}

DOI: 10.1103/PhysRevResearch.1.033046

\section{INTRODUCTION}

Light-matter interaction (LMI) is modified by interfacing the active material with an optical cavity or a plasmonic resonator. In the strong-coupling regime, the electronic transition dipole and the resonator exchange energy faster than they dissipate it, generating hybrid quasiparticles such as cavity polaritons or plexcitons [1-6]. These quasiparticles have been studied in the nonequilibrium regime, where photonic and optoelectronic devices operate [7-10]. To link their optical response directly to the underlying electron dynamics requires a quantitative model of their nonequilibrium behavior on the relevant femtosecond and picosecond timescales.

Exceptionally strong LMI combined with strong nonlinear optical response [11-13] and flexible processing and fabrication techniques brought semiconducting layered transition metal dichalcogenides (TMDs), in particular $\mathrm{MoS}_{2}$ and $\mathrm{WS}_{2}$,

\footnotetext{
*Corresponding author: bojana.visic@ipb.ac.rs

$\dagger$ christoph.gadermaier@polimi.it
}

Published by the American Physical Society under the terms of the Creative Commons Attribution 4.0 International license. Further distribution of this work must maintain attribution to the author(s) and the published article's title, journal citation, and DOI. into the focus of optoelectronics, nanophotonics, and energy harvesting research [14-21]. Strong excitonic resonances, especially the $A$ and $B$ resonances associated with direct transitions between the two spin-orbit split valence band (VB) maxima and the conduction band (CB) minimum at the $K$ point [22] dominate their optical spectra. In microcavities [23-25] or waveguides [26,27], single- and few-layer TMDs exhibit exciton-polaritons originating from strong coupling. Besides the two-dimensional platelets, TMDs also form fullerenelike structures and nanotubes (NTs) [28-41]. In a suitable geometry the high refractive index of TMDs provides an intrinsic optical cavity mode (CMR) that strongly couples to the excitonic resonance of nanotubes [42,43] and nanodisks [44].

Here, we provide a time-dependent study of the coupling between exciton resonances and an intrinsic optical CMR. We use femtosecond (fs) broadband optical pump-probe spectroscopy to examine the response of $\mathrm{WS}_{2} \mathrm{NTs}$ in aqueous dispersion and model the time-dependent extinction spectra with a simple phenomenological coupled oscillator (PCO) model [42] with a minimum set of time-dependent parameters. The temporal evolution of the model parameters reveals the nonequilibrium electron dynamics and associated manybody effects. The proposed model is applicable to numerous other material systems where an optical or plasmonic resonance couples to one or few electronic dipole transitions and 


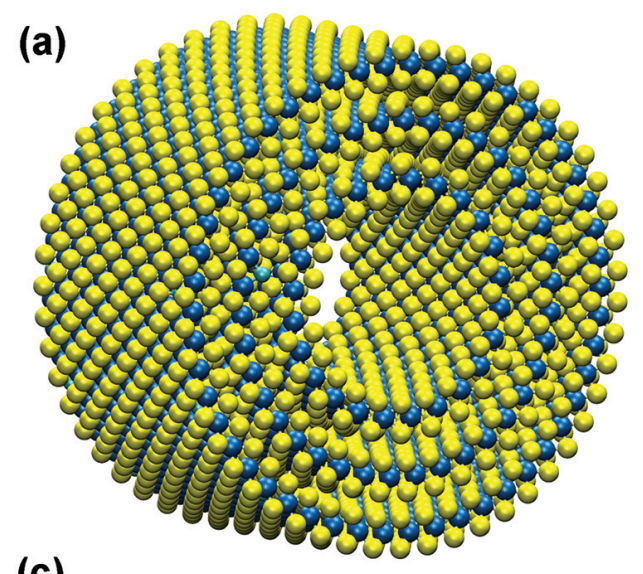

(c)

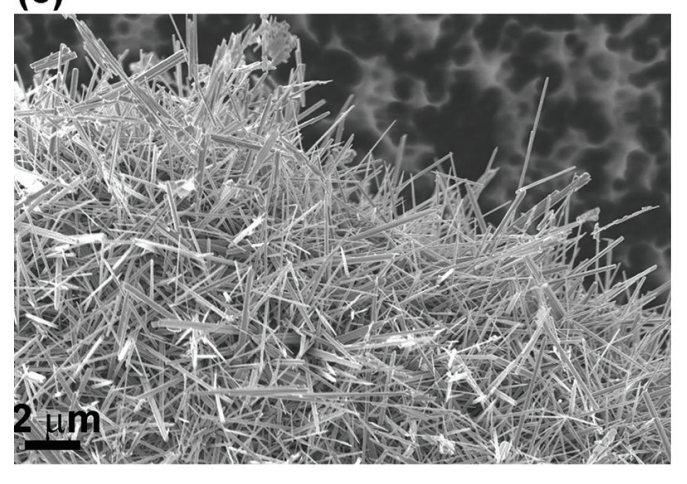

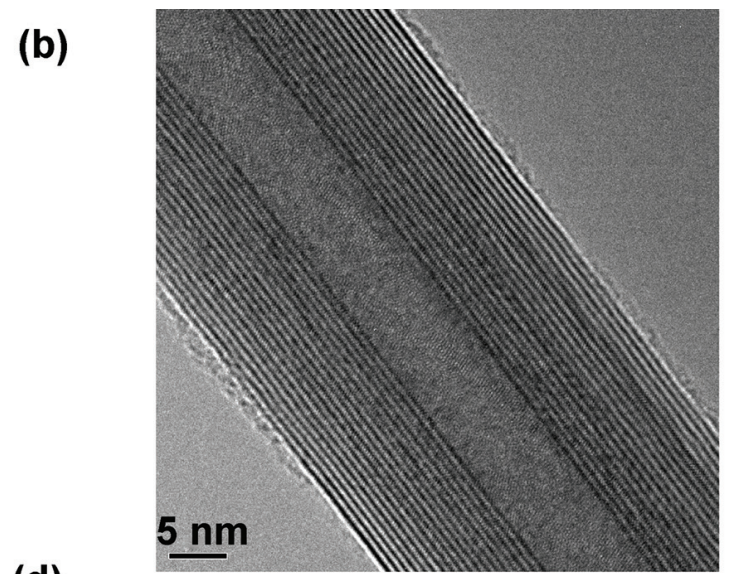

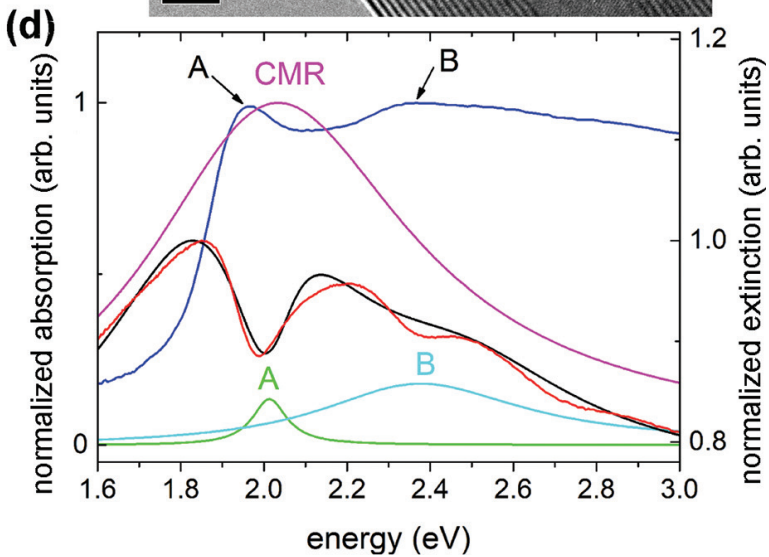

FIG. 1. (a) A schematic representation of a three-walled $\mathrm{WS}_{2}$ NT. (b) Typical transmission electron micrograph and (c) scanning electron micrograph of $\mathrm{WS}_{2}$ NTs. (d) Normalized pure absorbance (blue) and extinction (red) spectra of $\mathrm{WS}_{2}$ NT presented together with a PCO fit (black) and its constituent peaks ( $A$, green; $B$, cyan, and CMR, pink).

will yield valuable insight into the nonequilibrium behavior of polaritons and plexcitons.

\section{RESULTS AND DISCUSSION}

The $\mathrm{WS}_{2}$ NTs, schematically represented in Fig. 1(a) have 20-40 concentric shells, an average diameter of 40-120 nm, and 1-10 $\mu \mathrm{m}$ length [35]. Transmission and scanning electron microscopy (TEM and SEM) images are presented in Figs. 1(b) and 1(c).

\section{A. Equilibrium optical properties}

The absorption and extinction spectra of $\mathrm{WS}_{2}$ NTs in aqueous dispersion have distinctly different profiles, shown in Fig. 1(d). Absorption shows the prominent $A, B$, and $C$ resonances at 1.97, 2.38, and $2.76 \mathrm{eV}$ [45], originating from electronic transitions $A^{0}, B^{0}$, and $C^{0}$ between neutral ground states and excitons, as well as doping-induced transitions $A^{ \pm}$ and $B^{ \pm}$from charged ground states towards trions [46]. The $A$ and $B$ peaks as well as the CMR, obtained from the modeling described below, are also shown in Fig. 1(d). In extinction, the strong coupling of the excitonic transitions to the CMR yields three peaks at $1.85,2.21$, and $2.48 \mathrm{eV}$ [42], separated by transparency dips roughly at the positions of the $A$ and $B$ absorption peaks. Generally, the extinction spectrum of two coupled oscillators, such as CMR- $A$ or CMR- $B$, shows two peaks that are shifted away from each other compared to the resonance energies of the constituent oscillators. This shift increases with increasing coupling strength and with decreasing energy separation between the resonators. The extinction spectrum of the $\mathrm{WS}_{2}$ NTs shows one peak that is redshifted compared to the electronic $A$ resonance and one that is blueshifted relative to the $B$ resonance due to their coupling to the CMR. Since the CMR is much closer to $A$ than to $B$, the $A$-CMR repulsion dominates and the middle extinction peak appears blueshifted compared to the CMR.

We model the extinction spectrum using a PCO model consisting of three coupled Lorentzian oscillators: the CMR and the $A$ and $B$ resonances [42].

$$
C_{\text {ext }}(\omega) \alpha \omega \operatorname{Im}\left\{\frac{D_{A}(\omega) D_{B}(\omega)}{D_{\mathrm{CMR}}(\omega) D_{A}(\omega) D_{B}(\omega)-4 \omega_{A}^{2} g_{A}^{2} D_{B}(\omega)-4 \omega_{B}^{2} g_{B}^{2} D_{A}(\omega)}\right\} .
$$

Here, $g_{A}$ and $g_{B}$ are the couplings of the CMR to $A$ and $B$, and $D_{i}=D_{i}(\omega)=\omega_{i}^{2}+i \omega_{i} \gamma_{i}-\omega^{2}$, with $\omega_{i}$ the frequencies and $\gamma_{i}$ the widths of the respective resonances. Due to the high refractive index of the NTs, their intrinsic cavity efficiently 
TABLE I. The data derived from the PCO model fit to the measured extinction of the $\mathrm{WS}_{2}$ nanotubes.

\begin{tabular}{|c|c|c|c|c|c|c|c|}
\hline \multicolumn{3}{|c|}{$\begin{array}{c}\bar{h} \omega(\mathrm{eV}) \\
{[\text { Wavelength }(\mathrm{nm})]}\end{array}$} & \multicolumn{3}{|c|}{$\begin{array}{c}\gamma(\mathrm{eV}) \\
\text { [apparent damping frequency }(\mathrm{Hz}), \text { i.e., linewidth] }\end{array}$} & \multicolumn{2}{|c|}{$\begin{array}{c}g(\mathrm{eV}) \\
{[\text { coupling strength }(\mathrm{Hz})]}\end{array}$} \\
\hline CMR & Exciton $A$ & Exciton $B$ & CMR & Exciton $A$ & Exciton $B$ & With exciton $A$ & With exciton $B$ \\
\hline $\begin{array}{l}2.03 \\
(611)\end{array}$ & $\begin{array}{l}2.01 \\
(617)\end{array}$ & $\begin{array}{c}2.38 \\
(521)\end{array}$ & $\begin{array}{c}0.76 \\
\left(1.16 \times 10^{15}\right)\end{array}$ & $\begin{array}{c}0.10 \\
\left(1.52 \times 10^{14}\right)\end{array}$ & $\begin{array}{c}0.59 \\
\left(8.87 \times 10^{14}\right)\end{array}$ & $\begin{array}{c}0.13 \\
\left(1.98 \times 10^{14}\right)\end{array}$ & $\begin{array}{c}0.18 \\
\left(2.74 \times 10^{14} \mathrm{~Hz}\right)\end{array}$ \\
\hline
\end{tabular}

captures the incident light, greatly enhancing its intensity inside the cavity. Excitation of the $A$ and $B$ resonances via the coupling to the CMR is much stronger than direct excitation via the incident light. In Eq. (1) we neglect direct excitation of $A$ and $B$ and assume that the coupled oscillators interact with the incident light only via the CMR. Light leakage is a damping mechanism for the cavity and a scattering contribution to the extinction spectrum. If there were only the CMR without any absorption, the extinction of the NTs would be given only by this scattering contribution. In fact, upon setting $g_{A}=g_{B}=0$, Eq. (1) reduces to the single Lorentzian of the CMR. Equation (1) contains neither densities of absorbers nor oscillator strengths, hence it models the shape, but not the magnitude of the extinction spectrum.

The simple PCO model in Eq. (1) reproduces the extinction spectrum of the NT dispersion remarkably well, using the parameters listed in Table I, obtained from a least-square fit of Eq. (1) to the normalized extinction spectrum plotted in Fig. 1(d). The fit parameters are the resonance frequencies $\omega_{i}$ and widths $\gamma_{i}$ of the three Lorentzians and the couplings $g_{A, B}$ of $A$ and $B$ to the CMR. We also plotted the three Lorentzians in Fig. 1(d). Since no fit parameter is linked directly to their height, we normalized the CMR to height 1 and $A$ and $B$ to 0.13 and 0.18 , respectively, which is $g_{A, B}$ expressed in $\mathrm{eV}$, as a guide to the eye that indicates their coupling strength to the CMR. The obtained widths and positions of $A$ and $B$ also agree very well with the absorption peaks.

Equation (1) describes the strong-coupling regime, where the coupling between two resonances exceeds both their dampings. The homogeneous linewidth associated with the damping of the $A$ exciton in semiconducting TMDs at room temperature is a few tens of $\mathrm{meV}[47,48]$. The $A$ and $\mathrm{B}$ peaks are inhomogeneously broadened due to the polydispersity of the NTs. Moreover, they consist of overlapping exciton and trion resonances. The CMR energy shifts over several hundred meV when changing the NT diameter from 40 to $100 \mathrm{~nm}$ [42]. Therefore, the CMR width should be largely determined by the polydispersity of the NT diameters and much less by its damping. It is hence reasonable that, despite their large linewidths obtained from the PCO fit, all three resonances have dampings smaller than the coupling of either resonance to the CMR, thus justifying the PCO model in the strongcoupling regime. The parameters $\gamma_{i}$ are an apparent damping, which is much larger than the actual damping. While no values for the coupling $g_{A, B}$ are available for TMDs coupled to external optical or plasmonic resonators [23-27], the depths of the transparency dips in those systems are comparable to those of our NTs, suggesting similar coupling strength.

The simple PCO model reproduces the extinction spectrum very well and we attribute the small deviations to the NT polydispersity. The mean CMR position is very close to $A$, but more than $2 g_{B}$ away from $B$, resulting in a strong transparency $\operatorname{dip}$ between $A$ and the CMR, but no dip between the CMR and $B$ in the model curve. However, due to the polydispersity of their diameters, some NTs should have a CMR much closer to their $B$ resonance, yielding the measured small transparency dip and the blueshifted extinction peak compared to the $B$ absorption resonance.

\section{B. Nonequilibrium femtosecond dynamics}

To elucidate the temporal dynamics of this strongly coupled system, we employ femtosecond broadband optical pump-probe spectroscopy. The sample is photoexcited by a femtosecond laser pulse (the pump) with a photon energy of $3.1 \mathrm{eV}$ and the transient transmission spectrum is measured with a second (lower fluence and broadband) femtosecond laser pulse (the probe) at a variable delay $t$ after the pump. The pump fluence of $40 \mu \mathrm{J} \mathrm{cm}^{-2}$ is about half the saturation fluence (see Supplemental Material Fig. S6 at [49]), which is much lower than in TMD flakes due to the efficient light capturing by the CMR. The relative change in transmission $\Delta T / T$, for $\Delta T / T \ll 1$, approximates

$$
\frac{\Delta T}{T}(\omega, t)=-\Delta C_{\mathrm{ext}}(\omega, t)=C_{\mathrm{ext}}(\omega,-)-C_{\mathrm{ext}}(\omega, t)
$$

with $C_{\text {ext }}(\omega, t)$ the time-dependent nonequilibrium extinction spectrum after, and $C_{\text {ext }}(\omega,-)$ the equilibrium extinction before photoexcitation.

The $\Delta C_{\text {ext }}(\omega, t)$ spectra are shown in Fig. 2(a) for selected delays and as a contour plot in Fig. 2(b). Their main features are two enhanced transmission [photobleaching (PB)] and two photoinduced absorption (PA) peaks. The PB peaks at the shortest delays are at 1.88 and $2.25 \mathrm{eV}$ and blueshift by $40 \mathrm{meV}$ during the first $30 \mathrm{ps}$. Similarly, the PA peaks at 1.98 and $2.38 \mathrm{eV}$ blueshift by 30 and $20 \mathrm{meV}$, respectively. Subsequently, the signal decays without further shifts or other changes in the spectral shape. For each transparency dip there is a PA with a redshifted PB, suggesting that photoexcitation flattens the dips and broadens them towards lower energies. The temporal evolution of $\Delta C_{\text {ext }}(\omega, t)$ at selected probe energies (at the PA and PB peaks) is shown in Fig. 2(c). The contour plot in Fig. 2(d) of the data obtained from the model described below shows the remarkable agreement with the experimental data.

\section{Modeling the transient extinction spectra}

The equilibrium extinction spectrum of the $\mathrm{WS}_{2} \mathrm{NTs}$ $\left[C_{\text {ext }}(\omega,-)\right.$ on the right side of Eq. (2)], is well reproduced by the PCO model [Eq. (1)]. Photoexcitation induces small 
(a)

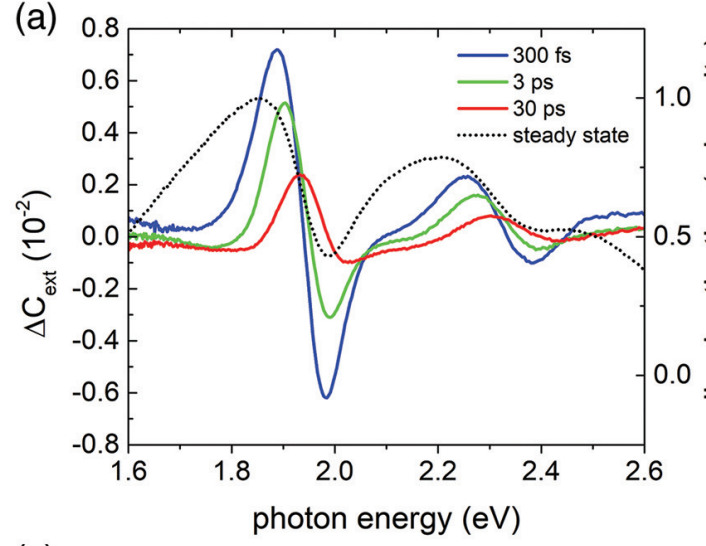

(c)

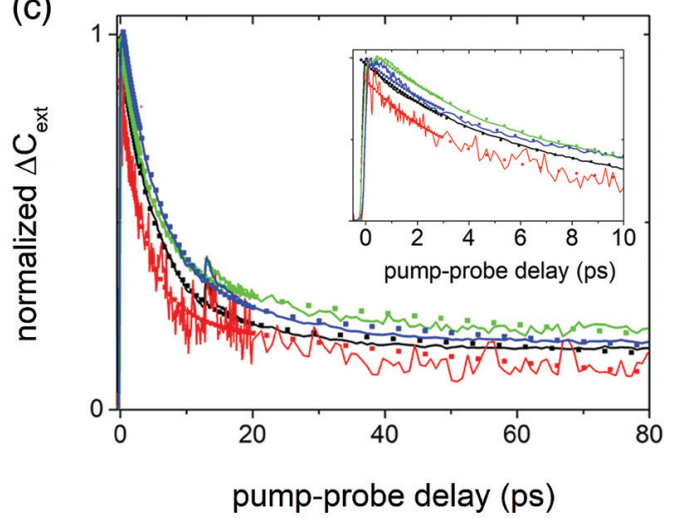

(b)

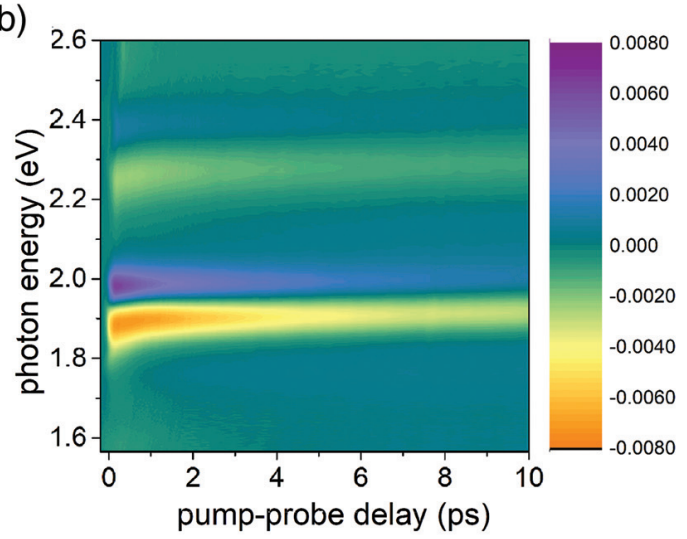

(d) 2.6

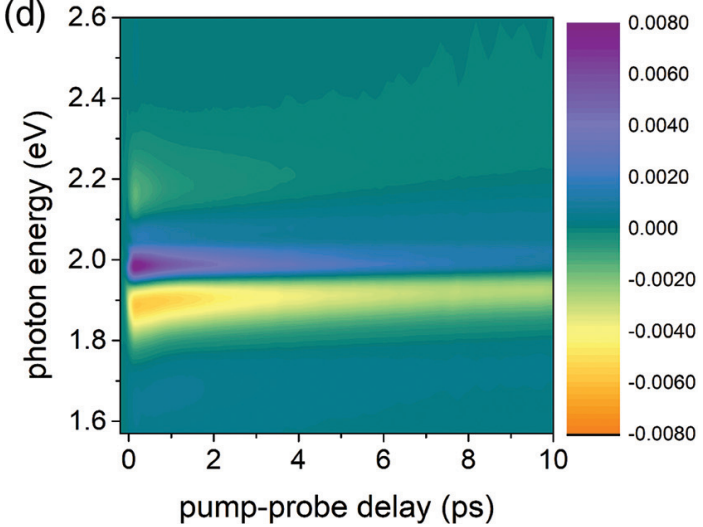

FIG. 2. (a) Differential extinction spectra $\Delta C_{\text {ext }}$ for the pump fluence of $40 \mu \mathrm{J} / \mathrm{cm}^{2}$ at delays $300 \mathrm{fs}$ (blue), $3 \mathrm{ps}$ (green), and $30 \mathrm{ps}$ (red) shown together with the steady-state extinction spectrum (black dotted line). (b) Contour plot of the measured $\Delta C_{\mathrm{ext}}$. (c) Normalized time traces for probe energies matching the equilibrium positions of the $A$ and $B$ excitons (1.99 and $2.39 \mathrm{eV}$, black and red lines, respectively) and the positions of two PB peaks (1.88 and $2.24 \mathrm{eV}$, blue and green lines, respectively), together with the biexponential function fit. Inset: zoom of the first 10 ps. (d) Contour plot of the calculated $\Delta C_{\text {ext }}$.

time-dependent changes to this spectrum. We model the nonequilibrium extinction spectrum $C_{\text {ext }}(\omega, t)$ in Eq. (2) with Eq. (1), with some of the parameters depending on $t$. In the Supplemental Material Fig. S1 [49], we describe the procedure step by step; here we briefly discuss the main results. We find that the only parameters that change appreciably upon photoexcitation and hence depend on $t$ are $\omega_{A}, g_{A}$, and, most strongly, $\gamma_{A}$ (see Fig. S2).

To rationalize the strong changes in $\gamma_{A}$ (see Supplemental Material Fig. S2), we note that $A$ consists of two overlapping resonances: the exciton peak $A^{0}$ and the redshifted trion peak $A^{ \pm}$. To obtain more physically meaningful parameters, we developed a PCO model with separate $A^{ \pm}$and $A^{0}$ peaks (see Supplemental Material Fig. S3 [49]). The fit of $\Delta C_{\text {ext }}(\omega, t)$ is shown as a contour plot in Fig. 2(d). The simple time-dependent PCO model reproduces the transient spectra remarkably well, especially below $2.1 \mathrm{eV}$, where the $A$-CMR coupling dominates. Only $A^{ \pm}$and $A^{0}$ change with time, suggesting that the coupling between the CMR and the relatively distant $B$ resonance is less relevant for the changes of the extinction. Our simple PCO model approximates the polydisperse NT ensemble with one broad CMR. A subset of the ensemble with a CMR much closer to $B$ may be sensitive also to time-dependent changes of the $B$ parameters; hence the small signal above $2.1 \mathrm{eV}$ is modeled less precisely.
The time-dependent coupling strength, center energy, and apparent damping of $A^{ \pm}$and $A^{0}$ are shown in Fig. 3. Immediately upon photoexcitation, $g_{\text {trion }}$ rises sharply, while $g_{\text {exciton }}$ decreases [Fig. 3(a)]. Since the electronic resonances couple to the CMR via absorption and emission of photons, the coupling strength is proportional the density of absorbers/emitters. Photoexcitation creates electrons and holes which act as (photoinduced) absorbers for the $A^{ \pm}$transition and diminish the available states for the transition from neutral ground states to excitons, thus enhancing $A^{ \pm}$at the expense of $A^{0}$.

Besides the change in the coupling strength, straightforwardly linked to the photoexcited state populations, also the resonance energies [Fig. 3(b)] and widths [Fig. 3(c)] of $A^{ \pm}$ and $A^{0}$ change during the electron relaxation. The $A^{ \pm}$and $A^{0}$ energies depend on the charge density in the sample, as the consequence of two antagonistic many-body effects [50,51]: band-gap renormalization redshifts the optical resonances, while screening reduces the exciton binding energy, causing a blueshift. The two effects almost cancel each other, with a slight prevalence of the blueshift for $A^{0}$ and redshift of $A^{ \pm}$for increased negative charge density observed in a monolayer $\mathrm{WS}_{2}$ transistor upon electrical gating. We expect a similar shift of $A^{ \pm}$and $A^{0}$ from the photoinduced generation of charges in our experiment $[52,53]$. However, no quantitative 
(a)

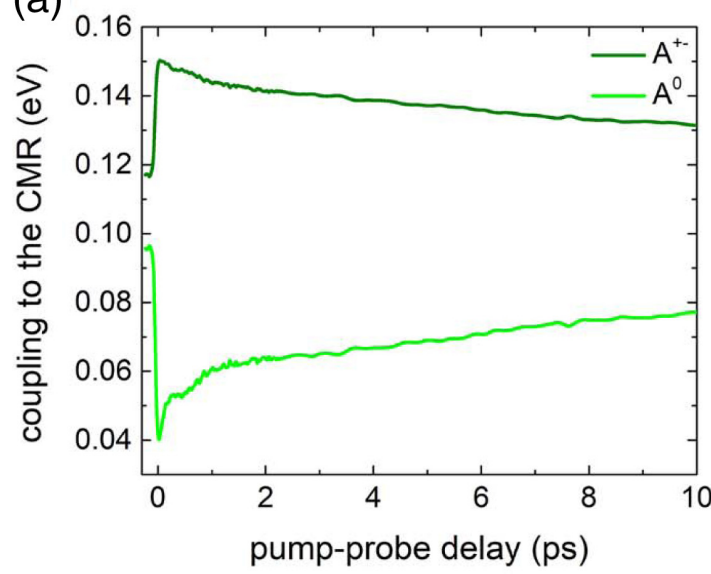

(c)

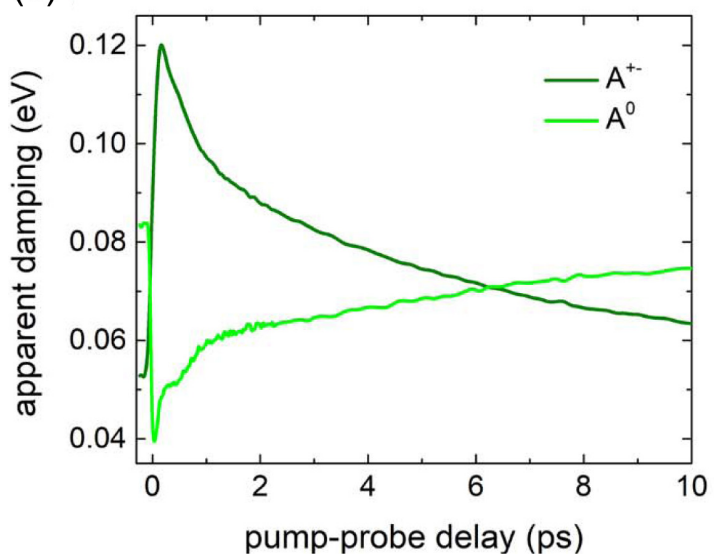

(b)

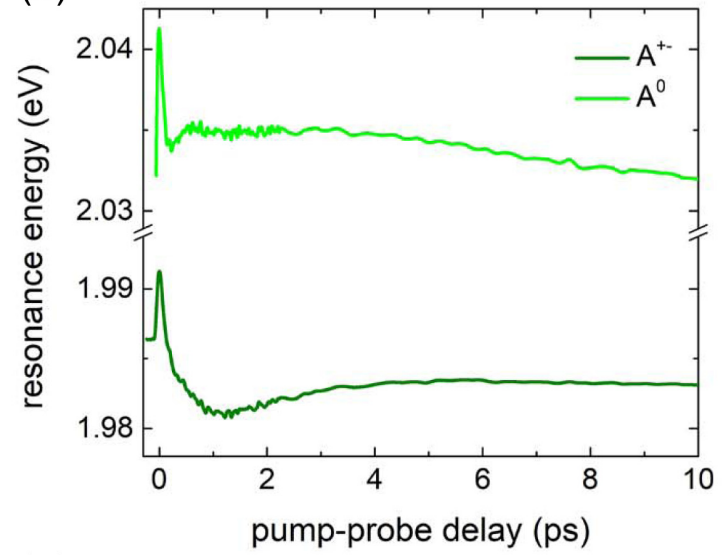

(d)

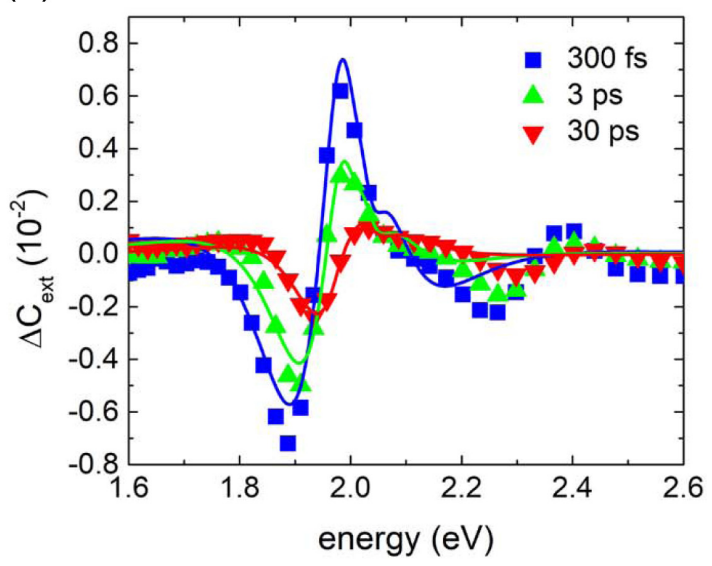

FIG. 3. Time evolution of fit parameters of the CMR model: (a) $A^{ \pm}$trion and $A^{0}$ exciton coupling to the CMR, (b) resonance energies, and (c) apparent damping. (d) Measured (symbols) and fitted (line) $\Delta C_{\text {ext }}$ spectra for selected pump-probe delays.

prediction of the imbalance between the two effects exists. The resulting shift of $A^{ \pm}$and $A^{0}$ upon photodoping may be different for different NTs within our distribution, both in sign and magnitude [54], thus changing both the energies and widths of the resonances. Moreover, for electrical doping, charge accumulates in the $\mathrm{CB}$ minimum [50], while photoexcitation at $3.1 \mathrm{eV}$, close to the $C$ exciton resonance, primarily populates a band nesting region far away from the $\mathrm{CB}$ and VB extrema [55]. As the photoexcited electrons and holes relax within the bands, we expect their contribution to both the band-gap renormalization and the screening of the exciton binding energy to change. Therefore, while we cannot determine which processes cause redshifts or blueshifts as well as narrowings or broadenings of $A^{ \pm}$and $A^{0}$, we can link their time dependence to the electron and hole relaxation processes.

The femtosecond optical response has been studied for platelike layered TMDs, including $\mathrm{WS}_{2}$, which lack an intrinsic cavity [56-73]. For these materials, photoexcitation with energy significantly above the $A$ and $B$ resonances creates hot electron-hole pairs. The hot carriers thermalize on a sub100 fs timescale, relax towards the band extrema with a time constant $\tau$ around $1 \mathrm{ps}$, and diffuse to get trapped or coalesce into indirect excitons with $\tau$ of a few picoseconds [56-58]. In the Supplemental Material Fig. S3 [49], we show fits of the temporal evolution of all time-dependent PCO parameters. They consistently show $\tau_{1}=60 \mathrm{fs}, \tau_{2}=540 \mathrm{fs}$, and $\tau_{3}=8.3 \mathrm{ps}$, suggesting the same relaxation mechanisms for $\mathrm{WS}_{2}$ NTs and platelets. Since the NT circumference is much larger than the exciton radius, quantum confinement due to the wrapping into a NT is negligible and excitons and trions behave like in two-dimensional TMD platelets. The relaxation dynamics of coupled oscillators is dominated by the subsystem with the faster relaxation pathways. Excitons and trions relax faster than the CMR damping, confirming the high quality of the NTs' intrinsic optical cavity.

\section{METHODS}

The multiwall $\mathrm{WS}_{2}$ nanotubes used in the current work were purchased from Nano-Materials Ltd. and were synthesized as pure phase according to the procedures published in [35]. The synthesis was carried out at 800-950 ${ }^{\circ} \mathrm{C}$ using a mixture of different phases of $\mathrm{WO}_{x}(2.83 \leqslant x \leqslant 3)$ as precursors. The $\mathrm{WS}_{2}$ nanotubes used in this work are $40-100 \mathrm{~nm}$ in diameter, with 20-40 concentric shells, and are 1-20 $\mu \mathrm{m}$ long, as shown in Fig. 1(a).

The solutions for all the measurements were prepared using purified water (Milli-Q RG, Millipore; $18 \mathrm{M} \Omega$ ). The mixture was hand-shaken and subsequently sonicated twice for 
1-3 min using ultrasonic bath. This mild sonication procedure does not significantly affect the nanotubes, as demonstrated by SEM and TEM analyses. All suspensions were measured using quartz cuvettes. UV-vis extinction measurements were carried out using a Cary-5000 spectrometer (Varian). Samples were prepared by adding $0.6 \mathrm{mg}$ of the $\mathrm{WS}_{2}$ nanotubes into $9 \mathrm{ml}$ of water. In order to separate the scattering and the absorbance processes from the total extinction spectra, decoupled absorption spectra were measured using a Hamamatsu Quantaurus absolute quantum yield system. This setup allows for the direct measurement of the amount of absorbed light, by placing the sample inside an integrating sphere. The system was calibrated using a sample with known absorbance to extract the optical absorbance.

The femtosecond pump-probe spectroscopy setup is driven by an amplified Ti:sapphire laser (Coherent Libra 2) producing $4 \mathrm{~W}$, sub-100 fs, $800 \mathrm{~nm}$ pulses at $2 \mathrm{kHz}$ repetition rate. A fraction of the pulse energy is frequency doubled in a nonlinear crystal, serving as the pump pulse. Another fraction of the pulse energy is focused in a 3-mm-thick sapphire plate to generate a single filament white light continuum covering the visible region (1.6-2.6 eV) used as a probe. Pump and probe are noncollinearly focused on the sample and the transmitted probe spectrum is detected by a spectrometer working at the full $2 \mathrm{kHz}$ repetition rate of the laser. $\Delta T / T$ spectra are recorded with a time resolution of sub-100 fs given by the cross-correlation of pump and probe pulses and a sensitivity of $1-2 \times 10^{-5}$. Pump and probe spot sizes of $0.3 \mathrm{~mm}$ are estimated with a beam profiler. The femtosecond pump-probe measurements were carried out for pristine $\mathrm{WS}_{2}$ nanotubes dispersed in $\mathrm{H}_{2} \mathrm{O}(0.15 \mathrm{mg}$ per $\mathrm{ml})$. The dispersions were sonicated twice for $2 \mathrm{~min}$ before the measurement itself.

\section{CONCLUSIONS}

We have reported on the ultrafast nonequilibrium LMI in $\mathrm{WS}_{2}$ nanotubes in aqueous dispersion, providing a timedependent study of the coupling between exciton resonances and an intrinsic cavity mode. We have developed a time- dependent PCO model that reproduces the transient extinction spectra and traced their time dependence back to the exciton and trion resonances, which are shifted due to photoinduced many-body effects. The modeling approach is applicable far beyond this example and can offer deeper insight also into the relaxation dynamics of cavity polaritons and plexcitons in numerous systems. Additionally, the model yields the coupling energy as a figure of merit for comparing different material/resonator combinations. The intrinsic cavity enhances the already remarkable LMI in TMD nanomaterials and makes $\mathrm{WS}_{2}$ NTs particularly interesting for nonlinear optics, photovoltaics, and optoelectronic devices. The transient spectra of the $\mathrm{WS}_{2} \mathrm{NTs}$, although dominated by the coupling of the exciton resonances to the CMR, can be traced back to the electron dynamics in the semiconductor, which is very similar to that observed in their layered counterparts. Future studies of optoelectronic and photonic devices based on TMD NTs can tap into the solid knowledge base available for the nonequilibrium optical properties of layered TMDs.

\section{ACKNOWLEDGMENTS}

We acknowledge the stimulating discussion with M. Celebrano and P. Biagioni, Ron Tenne for the work on the steadystate PCO model, and thank N. Zibouche for Fig. 1(a). The research leading to these results has received funding from the Marie-Curie ITN "MoWSeS" (Grant No. 317451); the Slovenian Research Agency programs P1-0040 and P1-0099; Serbian Ministry of Education, Science and Technological Development under Project No. III45018; the European Research Council advanced grant TRAJECTORY; and the European Union's Horizon 2020 research and innovation programme under Grant Agreement No. 654148 Laserlab-Europe. G.C. acknowledges support by the European Union Horizon 2020 Programme under Grant Agreement No. 785219 Graphene Core 2. This research was supported by the Israel Science Foundation Grant No. 265/12, the Perlman Family Foundation, and the Irving and Azelle Waltcher Foundation in honor of Professor M. Levy (Grant No. 720821).
[1] C. Weisbuch, M. Nishioka, A. Ishikawa, and Y. Arakawa, Phys. Rev. Lett. 69, 3314 (1992).

[2] J. Kasprzak et al., Nature (London) 443, 409 (2006).

[3] K. G. Lagoudakis, F. Manni, B. Pietka, M. Wouters, T. C. H. Liew, V. Savona, A. V. Kavokin, R. André, and B. DeveaudPlédran, Phys. Rev. Lett. 106, 115301 (2011).

[4] A. Amo et al., Science 332, 1167 (2011).

[5] W. Zhao et al., Adv. Mater. 28, 2709 (2016).

[6] B. G. DeLacy et al., Nano Lett. 15, 2588 (2015).

[7] M. Sukharev, T. Seideman, R. J. Gordon, A. Salomon, and Y. Prior, ACS Nano 8, 807 (2013).

[8] N. T. Fofang, N. K. Grady, Z. Fan, A. O. Govorov, and N. J. Halas, Nano Lett. 11, 1556 (2011).

[9] P. Vasa, R. Pomraenke, G. Cirmi, E. De Re, W. Wang, S. Schwieger, D. Leipold, E. Runge, G. Cerullo, and C. Lienau, ACS Nano 4, 7559 (2010).
[10] T. Schwartz, J. A. Hutchison, J. Léonard, C. Genet, S. Haacke, and T. W. Ebbesen, ChemPhysChem 14, 125 (2013).

[11] K. Wang et al., ACS Nano 7, 9260 (2013).

[12] H. Zhang, S. Lu, J. Zheng, J. Du, S. Wen, D. Tang, and K. Loh, Opt. Express 22, 7249 (2014).

[13] S. Zhang et al., ACS Nano 9, 7142 (2015).

[14] D. Lembke and A. Kis, ACS Nano 6, 10070 (2012).

[15] O. Lopez-Sanchez, D. Lembke, M. Kayci, A. Radenovic, and A. Kis, Nat. Nanotechnol. 8, 497 (2013).

[16] D. Vella et al., 2D Mater. 4, 021005 (2017).

[17] M.-L. Tsai, S.-H. Su, J.-K. Chang, D.-S. Tsai, C.-H. Chen, C.I. Wu, L.-J. Li, L.-J. Chen, and J.-H. He, ACS Nano 8, 8317 (2014).

[18] D. Voiry, M. Salehi, R. Silva, T. Fujita, M. W. Chen, T. Asefa, V. B. Shenoy, G. Eda, and M. Chhowalla, Nano Lett. 13, 6222 (2013). 
[19] K. F. Mak and J. Shan, Nat. Photonics 10, 216 (2016).

[20] Z. Sun, A. Martinez, and F. Wang, Nat. Photonics 10, 227 (2016).

[21] R. Wang, B. A. Ruzicka, N. Kumar, M. Z. Bellus, H.-Y. Chiu, and H. Zhao, Phys. Rev. B 86, 045406 (2012).

[22] R. Frindt and A. Yoffe, Proc. R. Soc. London, Ser. A 273, 69 (1963).

[23] X. Liu, T. Galfsky, Z. Sun, F. Xia, E.-c. Lin, Y.-H. Lee, S. KénaCohen, and V. M. Menon, Nat. Photonics 9, 30 (2015).

[24] L. C. Flatten, Z. He, D. M. Coles, A. A. P. Trichet, A. W. Powell, R. A. Taylor, J. H. Warner, and J. M. Smith, Sci. Rep. 6, 33134 (2016).

[25] S. Dufferwiel et al., Nat. Commun. 6, 33134 (2015).

[26] F. Hu, Y. Luan, M. E. Scott, J. Yan, D. G. Mandrus, X. Xu, and Z. Fei, Nat. Photonics 11, 356 (2017).

[27] Z. Fei, M. E. Scott, D. J. Gosztola, J. J. Foley, J. Yan, D. G. Mandrus, H. Wen, P. Zhou, D. W. Zhang, Y. Sun, J. R. Guest, S. K. Gray, W. Bao, G. P. Wiederrecht, and X. Xu, Phys. Rev. B 94, 081402(R) (2016).

[28] R. Tenne, L. Margulis, M. Genut, and G. Hodes, Nature (London) 360, 444 (1992).

[29] M. Hershfinkel, L. Gheber, V. Volterra, J. Hutchison, L. Margulis, and R. Tenne, J. Am. Chem. Soc. 116, 1914 (1994).

[30] Y. Feldman, E. Wasserman, D. Srolovitz, and R. Tenne, Science 267, 222 (1995).

[31] L. Yadgarov et al., Angew. Chem., Int. Ed. 51, 1148 (2012).

[32] W. Hsu et al., Chem. Mater. 12, 3541 (2000).

[33] I. Milosevic, B. Nikolic, E. Dobardzic, M. Damnjanovic, I. Popov, and G. Seifert, Phys. Rev. B 76, 233414 (2007).

[34] G. Seifert, H. Terrones, M. Terrones, G. Jungnickel, and T. Frauenheim, Phys. Rev. Lett. 85, 146 (2000).

[35] A. Zak, L. Sallacan-Ecker, A. Margolin, M. Genut, and R. Tenne, Nano 4, 91 (2009).

[36] B. Višić, L. S. Panchakarla, and R. Tenne, J. Am. Chem. Soc. 139, 12865 (2017).

[37] F. Qin, W. Shi, T. Ideue, M. Yoshida, A. Zak, R. Tenne, T. Kikitsu, D. Inoue, D. Hashizume, and Y. Iwasa, Nat. Commun. 8, 14465 (2017).

[38] M. Chhowalla and G. A. Amaratunga, Nature (London) 407, 164 (2000).

[39] L. Rapoport, Y. Feldman, M. Homyonfer, H. Cohen, J. Sloan, J. Hutchison, and R. Tenne, Wear 225, 975 (1999).

[40] M. Remskar, A. Mrzel, Z. Skraba, A. Jesih, M. Ceh, J. Demsar, P. Stadelmann, F. Levy, and D. Mihailovic, Science 292, 479 (2001).

[41] B. Visic, R. Dominko, M. K. Gunde, N. Hauptman, S. D. Skapin, and M. Remskar, Nanoscale Res. Lett. 6, 593 (2011).

[42] L. Yadgarov et al., Phys. Chem. Chem. Phys. 20, 20812 (2018).

[43] D. Kazanov et al., Appl. Phys. Lett. 113, 101106 (2018).

[44] R. Verre, D. G. Baranov, B. Munkhbat, J. Cuadra, M. Käll, and T. Shegai, Nat. Nanotechnol. 14, 679 (2019).

[45] C. Ballif, M. Regula, and F. Levy, Sol. Energy Mater. Sol. Cells 57, 189 (1999).

[46] K. F. Mak, K. He, C. Lee, G. H. Lee, J. Hone, T. F. Heinz, and J. Shan, Nat. Mater. 12, 207 (2013).
[47] G. Moody et al., Nat. Commun. 6, 8315 (2015).

[48] M. Selig, G. Berghäuser, A. Raja, P. Nagler, C. Schüller, T. F. Heinz, T. Korn, A. Chernikov, E. Malic, and A. Knorr, Nat. Commun. 7, 13279 (2016).

[49] See Supplemental Material at http://link.aps.org/supplemental/ 10.1103/PhysRevResearch.1.033046 for detailed timedependent PCO modeling and intensity dependence of the transient absorption signal.

[50] A. Chernikov, A. M. van der Zande, H. M. Hill, A. F. Rigosi, A. Velauthapillai, J. Hone, and T. F. Heinz, Phys. Rev. Lett. 115, 126802 (2015).

[51] K. Yao, A. Yan, S. Kahn, A. Suslu, Y. Liang, E. S. Barnard, S. Tongay, A. Zettl, N. J. Borys, and P. James Schuck, Phys. Rev. Lett. 119, 087401 (2017).

[52] E. A. Pogna et al., ACS Nano 10, 1182 (2016).

[53] P. D. Cunningham, A. T. Hanbicki, K. M. McCreary, and B. T. Jonker, ACS Nano 11, 12601 (2017).

[54] Y. Yomogida, Z. Liu, Y. Ichinose, and K. Yanagi, ACS Omega 3, 8932 (2018).

[55] G. Eda, H. Yamaguchi, D. Voiry, T. Fujita, M. W. Chen, and M. Chhowalla, Nano Lett. 11, 5111 (2011).

[56] T. Borzda et al., Adv. Funct. Mat. 25, 3351 (2015).

[57] V. Vega-Mayoral et al., Nanoscale 8, 5428 (2016).

[58] V. Vega-Mayoral et al., 2D Mater. 5, 015011 (2017).

[59] S. Sim, J. Park, J.-G. Song, C. In, Y.-S. Lee, H. Kim, and H. Choi, Phys. Rev. B 88, 075434 (2013).

[60] L. Yuan and L. Huang, Nanoscale 7, 7402 (2015).

[61] M. Massicotte, P. Schmidt, F. Vialla, K. G. Schädler, A. Reserbat-Plantey, K. Watanabe, T. Taniguchi, K.-J. Tielrooij, and F. H. Koppens, Nat. Nanotechnol. 11, 42 (2016).

[62] G. Kime, M. A. Leontiadou, J. R. Brent, N. Savjani, P. O’Brien, and D. Binks, J. Phys. Chem. C 121, 22415 (2017)

[63] P. D. Cunningham, K. M. McCreary, A. T. Hanbicki, M. Currie, B. T. Jonker, and L. M. Hayden, J. Phys. Chem. C 120, 5819 (2016).

[64] G. Aivazian, H. Yu, S. Wu, J. Yan, D. G. Mandrus, D. Cobden, W. Yao, and X. Xu, 2D Mater. 4, 025024 (2017).

[65] L. Wang et al., Nat. Commun. 8, 13906 (2017).

[66] D. Tsokkou, X. Yu, K. Sivula, and N. Banerji, J. Phys. Chem. C 120, 23286 (2016).

[67] T. Völzer, M. Lütgens, F. Fennel, and S. Lochbrunner, J. Phys. B: At. Mol. Opt. 50, 194003 (2017).

[68] C. Backes, A. Mitioglu, V. Vega-Mayoral, D. Hanlon, J. Coleman, V. Ivanov, D. Maude, and P. Plochocka, Nanotechnology 27, 425701 (2016).

[69] F. Ceballos and H. Zhao, Adv. Funct. Mater. 27, 1604509 (2017).

[70] H. Shi, R. Yan, S. Bertolazzi, J. Brivio, B. Gao, A. Kis, D. Jena, H. G. Xing, and L. Huang, ACS Nano 7, 1072 (2013).

[71] Z. Nie et al., ACS Nano 8, 10931 (2014).

[72] L. Wibmer, S. Lages, T. Unruh, and D. M. Guldi, Adv. Mater 30, 1706702 (2018).

[73] C. Ruppert, A. Chernikov, H. M. Hill, A. F. Rigosi, and T. F. Heinz, Nano Lett. 17, 644 (2017). 